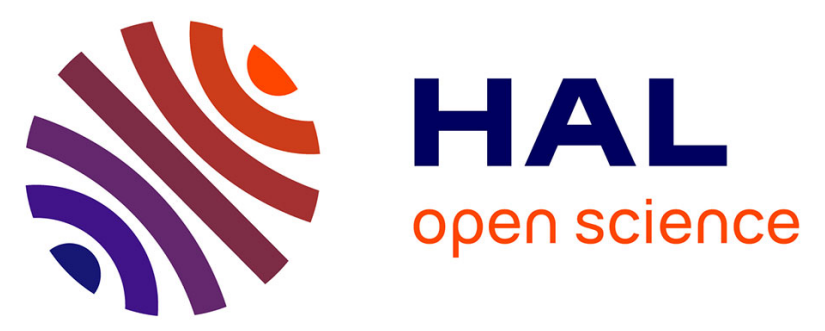

\title{
Effects of anti-glutamic acid decarboxylase antibodies associated with neurological diseases.
}

\author{
Mario-Ubaldo Manto, Marie-Aline Laute, Michèle Aguera, Véronique \\ Rogemond, Massimo Pandolfo, Jérome Honnorat
}

\section{- To cite this version:}

Mario-Ubaldo Manto, Marie-Aline Laute, Michèle Aguera, Véronique Rogemond, Massimo Pandolfo, et al.. Effects of anti-glutamic acid decarboxylase antibodies associated with neurological diseases.: GAD-Ab and cerebellar function. Annals of Neurology, 2007, 61 (6), pp.544-551. 10.1002/ana.21123 . inserm-00141558

\section{HAL Id: inserm-00141558 https://www.hal.inserm.fr/inserm-00141558}

Submitted on 7 Sep 2011

HAL is a multi-disciplinary open access archive for the deposit and dissemination of scientific research documents, whether they are published or not. The documents may come from teaching and research institutions in France or abroad, or from public or private research centers.
L'archive ouverte pluridisciplinaire HAL, est destinée au dépôt et à la diffusion de documents scientifiques de niveau recherche, publiés ou non, émanant des établissements d'enseignement et de recherche français ou étrangers, des laboratoires publics ou privés. 
EFFECTS OF ANTI-GAD ANTIBODIES ASSOCIATED WITH NEUROLOGICAL DISEASES

Mario-Ubaldo Manto ${ }^{1}$, Marie-Aline Laute ${ }^{1}$, Michèle Aguera ${ }^{2}$, Véronique Rogemond ${ }^{2}$, Massimo Pandolfo ${ }^{1}$, Jérome Honnorat ${ }^{2}$

${ }^{1}$ Laboratoire de Neurologie Expérimentale, Hôpital Erasme, ULB, Bruxelles, Belgium And the:

${ }^{2}$ INSERM, U842, Lyon, F-69372 France; Université Lyon 1, UMR-S842 Lyon, F-69003

France; Hospices Civils de Lyon, Neurologie B, Lyon, F-69003 France

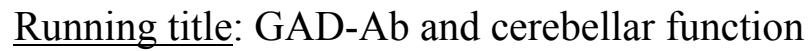

Word count for title: 8 words: (68 characters).

Word count for running title: 4 (30 characters).

Word count for the abstract: 203 words.

Word count for the text: 3418 words.

Number of references: 37

Number of tables: 1

Number of figures: 6 .

Supported by the Belgian National Research Foundation.

We declare no financial interest and we certify that the submission is not under review at any other publication.

All coauthors have seen and agree with the content of the manuscript.

Correspondence to: Professeur J. Honnorat,

Neurologie B, Hôpital Neurologique Pierre Wertheimer

59 boulevard Pinel,

69677 Bron cedex

e-mail: jerome.honnorat@chu-lyon.fr

phone: 33472357808

fax: 33472357329 
Objectives: Glutamic acid decarboxylase (GAD) catalyzes the conversion of glutamic acid into gamma-amino-butyric acid (GABA). GAD autoantibodies (GAD-Ab) have been described in diabetes mellitus and in diseases involving the central nervous system such as stiff-person syndrome and cerebellar ataxia. However, the pathogenic role of GAD-Ab in neurological diseases remains a matter of debate.

Methods: We analysed the effects of intra-cerebellar and paraspinal administration of GAD-Ab in rats using neurophysiological and neurochemical methods.

Results: Intra-cerebellar administration of IgG from patients with GAD-Ab and neurological involvement (IgG-GAD) blocked the potentiation of the corticomotor response normally associated with trains of repetitive peripheral nerve stimulation. When injected in the lumbar paraspinal region, IgG-GAD induced continuous motor activity with repetitive discharges, abnormal exteroceptive reflexes and increased excitability of anterior horn neurons, as assessed by F/M ratios. Furthermore, IgG-GAD significantly reduced the NMDA-mediated production of nitric oxide (NO) in cerebellar nuclei and impaired the synaptic regulation of glutamate following NMDA administration. These effects were not observed after administration of IgG from (a) patients with GAD-Ab, diabetes mellitus and without neurological complications, nor were they observed with (b) IgG from control patients..

Interpretation: These results indicate that stiff-person syndrome and cerebellar ataxia are the direct consequence of antibody-mediated neuronal dysfunction.

Key words: cerebellum; GAD-autoantibodies; nitric oxide; in vivo microdialysis; learning; spinal cord. 
Antibodies to glutamic acid decarboxylase (GAD-Ab) are considered to be markers of autoimmune type 1 diabetes mellitus because they are found in the serum of most type 1 diabetes patients even before clinical onset. ${ }^{1}$ GAD-Ab are also found in possibly immune-mediated syndromes affecting the central nervous system (CNS), including stiff-person syndrome ${ }^{2}$ and a small group of patients with cerebellar ataxia. ${ }^{3-4}$ The role of GAD-Ab in the pathogenesis of these neurological disorders is not known. Some authors argue that GAD-Ab might simply reflect the frequent association of these diseases with diabetes mellitus. There is evidence to the contrary, however: 1.some patients never develop diabetes, 2.the GAD epitopes recognized by sera from neurological patients are different from those recognized by sera from patients with diabetes mellitus alone. ${ }^{3}, 3$.GAD-Ab titers are significantly higher in the serum of neurological patients. Intrathecal synthesis has been demonstrated in a few patients with neurological involvement, suggesting a possible pathogenic role of GAD-Ab in neurological symptoms. ${ }^{3,5}$ In vitro, GAD-Ab from patients with neurological syndromes induce a suppression of gammaamino-butyric acid (GABA) release..$^{6-7}$ In a whole-cell patch-clamp model with rat cerebellar slices, GAD-Ab were shown to modulate inhibitory potentials in interneurons in a dosedependent manner and to down-regulate GABA synthesis in basket cells terminals, reducing GABA release on post-synaptic Purkinje cells. ${ }^{6}$ GAD-Ab may therefore impair neuronal activity and interfere with the regulation of brain neurotransmitters. However, the effects of GAD-Ab have not been studied in the intact animal thus far. We assessed the in vivo effects of GAD-Ab extracted from patients with or without neurological symptoms on: (1) the adaptation of the motor cortex to sustained peripheral stimulation, a model of learning requiring the integrity of the cerebellum, ${ }^{8-9}(2)$ the spontaneous motor activity in skeletal muscle, the exteroceptive reflexes and the $\mathrm{F} / \mathrm{M}$ ratios, ${ }^{10}$ (3) the NMDA-induced production of nitric oxide (NO) in the cerebellum, ${ }^{11}(4)$ the regulation of extra-cellular concentrations of glutamate following administration of NMDA in deep cerebellar nuclei.

\section{Methods}

Antibodies. Sera were collected from five patients with GAD-Ab and neurological syndromes (three with stiff-person syndrome and two with cerebellar ataxia). None of them had diabetes mellitus. Control sera were obtained from three patients with paraneoplastic cerebellar ataxia (one anti-Hu-positive; one anti-Yo-positive; one anti-CV2/CRMP5-positive), and from three patients with diabetes mellitus and GAD-Ab, but no neurological signs. All sera were stored at $-80^{\circ} \mathrm{C}$ before the experiments.

All experiments were performed with a purified IgG fraction. IgG were adsorbed to protein ASepharose beads (proteinA Sepharose 4 fast flow, Amersham Biosciences, France) and eluted with sodium citrate $(0.5 \mathrm{M}, \mathrm{pH} 2.5)$. After neutralization, samples were dialyzed overnight at $4^{\circ} \mathrm{C}$ against Ringer solution (Frenesius Kabi, France) and sterilized by filtration with $0.22 \mu \mathrm{m}$ filters. Protein concentration was measured by the Bradford method (Bio-Rad protein assay, Bio-Rad, France) and each sample was diluted or concentrated to obtain a protein level of $1 \mathrm{mg} / \mathrm{ml}$. The control solution was prepared with citrate buffer $(0.5 \mathrm{M}, \mathrm{pH} 2.5)$ neutralized and dialysed against Ringer solution in order to avoid osmotic drifts.

Animal studies were approved by the institutional animal care committee of the Free University of Brussels. Experiments were conducted in male Wistar rats (Charles River Laboratories; weight between 250 and $300 \mathrm{~g}$ ). See also table 1 for a summary of the experiments carried out. Microdialysis procedure. The day before the neurochemical investigations, animals were anesthetized for surgery using a loading dose of chloral hydrate given ip (400 mg/kg). The skull was exposed and an intra-cerebellar guide was implanted (coordinates of the extremity of the 
guide were AP: $-11.6, \mathrm{~L}:+2.3, \mathrm{~V}:-4.6$; all coordinates are related to bregma according to the atlas of Paxinos and Watson). The guide was fixed with dental cement and a probe was immediately inserted through the guide. A CMA/10 probe (length: $2 \mathrm{~mm}$, diameter: $0.5 \mathrm{~mm}$ ) was inserted in the left cerebellum using a stereotactic frame (accuracy $<0.1 \mathrm{~mm}$ ). The probe was connected to a microinfusion pump and perfused with Ringer solution at a flow rate of 2 $\mathrm{microL} / \mathrm{min}$. The rats were allowed to recover and were given free access to water and food. Twenty-four hours later, another loading dose of chloral hydrate $(400 \mathrm{mg} / \mathrm{kg}$ ip) was administered, followed by continuous ip infusion at a rate of $2 \mathrm{microL} / \mathrm{min}$. A volume of 5 microL of solution (control solution or solution with $\mathrm{Ab}$ ) was injected over a period of 5 minutes through the guide. The probe was then reinserted in the guide (CMA12, Carnegie Medicin, Sweden) and continuously perfused with a Ringer's solution (containing $148 \mathrm{mM} \mathrm{NaCl}, 1.1 \mathrm{mM}$ $\mathrm{CaCl} 2$ and $4 \mathrm{mM} \mathrm{KCl}, \mathrm{pH}+/-7.2$.) at a flow rate of $2 \mathrm{microL} / \mathrm{min}$.

All the animals underwent cerebellar surgery on the left side. At the end of the experiments, an overdose of chloral hydrate was administered and the brain was removed from the skull for histological verification of the location of the probe. ${ }^{8-10}$

Modulation of motor cortex excitability. We investigated the evoked responses in the left gastrocnemius muscle following stimulation of the right motor cortex, before (basal condition) and after repetitive electrical stimulation of the left sciatic nerve. ${ }^{8,12}$ Chloral hydrate was administered continuously at $2 \mathrm{microL} / \mathrm{min}$ (CMA100 micropump; CMA, Sweden) using the ip route. ${ }^{9}$ Anesthesia depth was adjusted for absence of abdominal contractions in response to tail pinch. The left sciatic nerve was surgically exposed for bipolar stimulation. Duration of stimulation was one hour. Trains of stimulation were delivered at a rate of $10 \mathrm{~Hz}$ (a train being composed of 5 stimuli of a 1 msec duration; A310-A365 stimulator - World Precision Instruments, UK). Stimulus intensity was adjusted to produce constant somatosensory evoked potentials (SEP) in the EEG. ${ }^{12}$ We specifically investigated the modulation of motor cortex excitability before and 1 hour after intra-cerebellar microinjection (see also the microdialysis procedure).

For the stimulation of the right motor cortex, square stimuli were applied via screws fixed on the skull at the level of the motor cortex. ${ }^{8}$ Peak-to-peak amplitudes in motor responses of the left gastrocnemius muscle were studied. Motor threshold was defined as the lowest intensity eliciting at least 5 out of 10 evoked responses with an amplitude $>20$ microVolts. The sigmoid feature of the recruitment curve was checked in each rat. ${ }^{12}$ The intensity of stimulation was $130 \%$ of motor threshold. ${ }^{9}$ Filter settings were $30 \mathrm{~Hz}-1.5 \mathrm{KHz}$ (NeuroMax 4, Xltek, Canada).

NO measurement. To assess NMDA-induced production of NO, experiments were conducted:

(1) in a control group of rats (Group I: $n=6$ ) injected in the cerebellum with a control solution,

(2) in a group of rats (Group II, $n=8$ ) injected in the cerebellum with IgG from patients with GAD-Ab and neurological symptoms, (3) in a second control group of rats injected in the cerebellum with IgG from patients with paraneoplastic cerebellar ataxia (anti-Yo Ab n =6; anti$\mathrm{Hu} \mathrm{Ab} \mathrm{n} \mathrm{=} \mathrm{6;} \mathrm{anti-CV2} \mathrm{Ab} \mathrm{n} \mathrm{=6).} \mathrm{We} \mathrm{assessed} \mathrm{also} \mathrm{the} \mathrm{effects} \mathrm{of} \mathrm{NMDA} \mathrm{on} \mathrm{the} \mathrm{extra-cellular}$ concentration of glutamate in six control rats and in five rats injected with IgG from GAD-Ab positive patients with neurological symptoms. Three baseline measurements of NO were performed in each rat to assess the variability in the basal condition. ${ }^{11}$ NMDA (concentration: 10 $\mathrm{mM}$ ) was administered in the probe and microdialysates were collected every 20 minutes (volume of each sample: 40 microL). NO concentrations were measured using a Nitric Oxide Quantitation Kit (Active Motif, Belgium) with a two-step assay method involving the addition of two cofactors to the nitrate reductase reaction. The reductase reaction was completed within 30 minutes and colorimetric determinations were directly measured by the addition of Griess Reagent, which converts nitrite into a purple-colored azo compound. Standard curves for both nitrate and nitrite were verified for each experiment. The absorbance was read on a spectrophotometer at $540 \mathrm{~nm}$ with a reference wavelength of $620 \mathrm{~nm}$. The validation of the 
method (stability of baseline measurements, as well as use of inhibitors of NO synthase such as L-NAME) has been published previously. ${ }^{11}$

Glutamate measurement. After stabilisation of baseline measurements, samples were collected every 20 minutes to measure extra-cellular concentrations of glutamate. We used the CMA/600 device (Aurora Borealis Control; glutamate oxidase method) for the quantitative determination of glutamate in microdialysates.

Spontaneous muscle activity. We investigated the spontaneous muscle activity in the left gastrocnemius muscle ( 5 recording sites) before ( 30 minutes) and after ( 45 minutes) the paraspinal administration of antibodies at the lumbar level. The spinal cord was surgically exposed using a microscope and a micro-injection of 10 microL (injected over a period of 10 minutes) was performed. A micromanipulator was used for the EMG study as reported previously. ${ }^{13}$ The spinal cord was subsequently dissected to check for the absence of local bleeding. In addition, both motor cortex stimulation and somatosensory evoked potentials (SEP) were used to monitor the absence of damage to the long tracts following the injection of antibodies.

F-waves and $M$ response. The $\mathrm{F}$-waves and the direct motor responses ( $\mathrm{M}$ response) were studied according to a method adapted from Gozariu et al. ${ }^{14}$ Electrical stimulation of the left tibial nerve was achieved through a pair of needle electrodes inserted subcutaneously at the ankle, behind the medial malleolus. Electrical stimuli consisted of single square-wave shocks of $0.5 \mathrm{msec}$ duration, delivered every 6 seconds. EMG recordings were obtained from the ispilateral plantaris muscle through a pair of needle electrodes inserted in the distal third of the sole (filters $30 \mathrm{~Hz}-1.5 \mathrm{KHz}$ ). We assessed the ratio mean $\mathrm{F} /$ mean $\mathrm{M}$ wave amplitudes following 50 supramaximal stimuli. ${ }^{8-9}$ These studies were first performed in the basal condition. As expected, Mean F/Mean M responses were similar in the 4 groups of rats investigated in the basal condition prior administration of antibodies (group 1 - controls: no administration of fluid; group 2: rats receiving saline administration; group 3: rats receiving IgG from patients with GAD-Ab and stiff-person syndrome or cerebellar ataxia; group 4: administration of GAD-Ab from patients without neurological symptoms). Mean values were in the range of 9 to $13 \%{ }^{8}$ We analysed these ratios 1 hour after administration of antibodies.

Exteroceptive reflexes were assessed by analysing the response of ipsilateral lower limbs following electrical stimulation of the sole with rectangular electrical stimuli (duration of pulses: $0.3 \mathrm{msec}$; intensity: 1 to $3 \mathrm{~mA}$ ). Subcutaneous needles were inserted proximally at the level of left gastrocnemius muscle and distally at the level of the left plantaris muscle.

\section{Statistical analysis}

The effects of repetitive peripheral stimulation on the amplitude of motor evoked potentials were evaluated in each of the 4 groups of rats (controls, administration of Ringer, administration of $\mathrm{GAD}-\mathrm{Ab}$ from patients with neurological symptoms, administration of GAD-Ab from patients with diabetes in absence of neurological symptoms) using the Student $t$ test. The mean F/mean M response ratio was compared among the 4 groups using the analysis of variance (ANOVA). The effects of antibodies on NMDA-induced production of NO and on regulation of glutamate concentrations by NMDA were evaluated by ANOVA, followed by multiple comparisons procedure. $^{15}$

\section{Results}

\section{Potentiation of the corticomotor response}

We investigated the evoked responses in the left gastrocnemius muscle following stimulation of the right motor cortex before (basal condition) and after repetitive electrical stimulation of the left sciatic nerve. Figure 1A shows the corticomotor responses in a control rat (no intracerebellar administration), in an animal injected with Ringer solution, in an animal injected with 
IgG from patients with GAD-Ab and neurological symptoms, and in a rat injected with IgG from patients with GAD-Ab and diabetes mellitus but no neurological signs. The enhancement of the corticomotor response is blocked when IgG from patients with GAD-Ab and neurological symptoms are injected. Baseline motor responses (before concurrent somatosensory stimulation) showed similar peak-to-peak values in the 4 groups (Figure 1B). In rats with no cerebellar injection, in rats with Ringer infusion and in rats injected with IgG from patients with diabetes mellitus, the cortico-muscular response was enhanced after repetitive stimulation of the sciatic nerve (stimulation effect: $\mathrm{p}<0.01$ in each group). By contrast, there was no enhancement of the peak-to-peak response when IgG from patients with GAD-Ab and neurological syndromes were administered into the cerebellar nuclei $(\mathrm{p}=0.18)$. Thus, potentiation was specifically impaired when rats were injected with IgG extracted from patients exhibiting neurological signs and GAD-Ab.

\section{Skeletal muscle/spinal cord function}

We investigated the spontaneous muscle activity in the left gastronemius muscle, before and after administration of IgG at the paraspinal lumbar level. As shown in figure 2, IgG from patients with GAD-Ab and neurological symptoms induced continuous motor activity in the gastrocnemius muscle, with repetitive discharges. Spontaneous small muscle twitches in lower limbs were observed in 5 of the 6 rats injected with GAD-Ab from neurological patients. However, no visible distortion of the spine was induced. Exteroceptive reflexes became abnormal with exaggerated proximal and distal responses of the ipsilateral limbs following electrical stimulation of the skin without evidence of long tract disturbance. In all the rats injected with IgG from neurological patients with GAD-Ab, stimulation of the sole resulted in visible contractions of the gastrocnemius muscle with bursts having a latency of 7-12 msec. This exaggerated response was absent in the other groups with no bursts of muscle activity in the left gastrocnemius muscle. F/M ratios increased one hour after IgG administration when antibodies from patients with neurological signs and GAD-Ab were used (Figure 3). IgG from patients with stiff-person syndrome and with cerebellar ataxia were equally effective. Again, no effect was found with IgG extracted from patients without neurological symptoms (analysis of variance, inter-group effect $\mathrm{p}<0.01$ ).

\section{NO and glutamate concentration}

We tested in vivo by microdialysis how GAD-Ab affected the glutamate/NO pathway in the cerebellum (Figure 4). As expected, in the control group administration of NMDA was associated with a significant increase in NO levels $(\mathrm{p}=0.002)$. In rats injected with IgG from patients with GAD-Ab and stiff-person syndrome or cerebellar ataxia, NO levels also significantly increased after NMDA administration $(\mathrm{p}=0.02)$. However, this increase was significantly less important than in controls (group by time interaction: $p=0.021$ ). Injection of IgG extracted from patients with paraneoplastic cerebellar ataxia did not impair the NMDAmediated production of NO as compared to controls $(\mathrm{p}=0.18)$. NO levels were significantly higher in the Hu-Ab, Yo-Ab and CV2/CRMP5-Ab groups as compared to the GAD-Ab group (group by time interaction: $\mathrm{p}<0.001$ ).

Finally, we explored the production of glutamate in the deep cerebellar nuclei as a complement to a previous study. ${ }^{6}$ As expected, infusion of NMDA in the cerebellar nuclei reduced the extracellular concentration of glutamate in control animals (Figure 5; $\mathrm{p}<0.001)$. Glutamate levels were significantly higher in rats injected with IgG from patients with GAD-Ab and neurological syndromes than in control groups (group by time interaction: $\mathrm{p}<0.001$ ). 


\section{Discussion}

Our results provide in vivo evidence with fairly acute studies that IgG purified from patients with GAD-Ab and neurological syndromes can alter cerebellar activity, impair learning and affect spinal cord activity in rodents. These effects were observed in two different experimental protocols with the antibodies altering both electrophysiology and neurochemistry in vivo. First, we assessed the increase in the cortical motor response normally induced by repeated somatosensory stimulation in rodents. ${ }^{12}$ We previously demonstrated that the cerebellum plays a key role in the modulation of this response, ${ }^{8-9}$ which involves an increased efficiency of the thalamo-cortical pathway. We also recently showed that the intra-cerebellar administration of tetrodotoxin, a sodium channel blocker, blocks the potentiation in the contralateral motor cortex response. This is considered the first step for learning in the paradigm of sustained peripheral stimulation. ${ }^{10}$ In the present study, we observed a similar effect after the intracerebellar injection of IgG from GAD-Ab positive patients with stiff-person syndrome or with cerebellar ataxia. We then tested whether the paraspinal administration of these antibodies could affect spinal cord function. Again, IgG isolated from patients with GAD-Ab and stiff-person syndrome or cerebellar ataxia induced repetitive muscle discharges, abnormal exteroceptive reflexes and increased F/M ratios, suggesting that these antibodies can enhance motoneuronal activity. IgG from GAD-Ab positive individuals and no CNS involvement were ineffective in both models. Recently, Sommer et al. described similar results with IgG from a patient with stiff-person syndrome and anti-amphiphysin antibodies. ${ }^{16}$ They showed that the injection of the IgG fraction of the patient's serum in rats resulted in a dose-dependent stiffness with spasms resembling those of human stiff-person syndrome. Taken together, our results and those of Sommer et al. strongly support that the stiff-person syndrome is directly caused by the effect of antibodies on spinal cord neurons, both in anti-amphiphysin and anti-GAD positive cases. However, IgG from GAD$\mathrm{Ab}$ positive patients with stiff-person syndrome and from patients with cerebellar ataxia cause the same types of dysfunction in the cerebellum and in the spinal cord, leaving unexplained why these patients develop typically distinct clinical pictures, although there have been some patients described with both syndromes. ${ }^{17-19}$ A few cases have been reported where, in patients with GAD-Ab, symptoms of stiff-person syndrome may improve with immunotherapy and IgGdepleting strategies, but symptoms of cerebellar dysfunction rarely improve. ${ }^{19-21}$ A possible explanation for this different response to treatment is that the cascade of events induced by antibodies differs in both conditions and the vulnerability of various sites in the CNS to GAD-Ab might differ. GAD-Ab acting upon cerebellar pathways might induce lesions reaching an irreversible stage, leading to neuronal destruction and cerebellar atrophy in a chronic situation. This hypothesis is supported by the recent publication of an autopsy of a patient with both cerebellar ataxia and stiff person syndrome showing only Purkinje cells loss and no abnormalities in the spinal cord. ${ }^{18}$

In cell and tissue culture systems, IgG from patients with neurological syndromes and GAD-Ab induce a suppression of GABA release, ${ }^{6-7}$ suggesting that they may change the balance between glutamate and GABA and cause glutamate excitotoxicity. To test this hypothesis in the living animal, we studied by microdialysis the effect of these IgG on glutamate and NO production. Our results demonstrate that GAD-Ab decrease NO production and impair the synaptic regulation of glutamate, resulting in increased concentrations of this neurotransmitter in the deep cerebellar nuclei. NO is an important signaling molecule involved in physiological processes such as brain development, synaptic plasticity, sensory processing, and motor learning. ${ }^{22}$ The glutamate/NO pathway is of primary importance in the cerebellum, ${ }^{23-24}$ where NMDA-induced production of NO is clearly involved in synaptic plasticity. ${ }^{25-28}$ Moreover, sustained inhibition of NO production induces apoptosis in differentiated cerebellar granules, while compounds which release NO significantly revert this effect. ${ }^{29}$ In brain synapses, NO synthase activation is mainly 
coupled to NMDA-mediated calcium entry at post-synaptic densities through regulatory protein complexes, and NMDA/NO inhibit the pre-synaptic release of glutamate. Accordingly, NO scavengers, NO blockers and inhibitors of NO synthase activity are known to impair motor behavior. ${ }^{30}$ Glutamate release inhibition also prevents excitotoxic neuronal death. ${ }^{31}$ We show that IgG from patients with GAD-Ab decrease NMDA-mediated NO production, a mechanism that may affect cerebellar activity and eventually lead to cell death.

Our results strongly support the hypothesis that antibodies directly cause stiff-person syndrome and cerebellar ataxia in individuals with GAD-Ab, but it remains to be determined whether the GAD-Ab themselves or other auto-antibodies contained in the purified IgG fraction from these patients are responsible. For example, recently some authors reported that antibodies against GABA-A receptor associated protein were also present in patients with stiff-person syndrome and GAD-Ab. ${ }^{32}$ The main arguments against a role of GAD-Ab are the intracellular localization of GAD and the fact that GAD-Ab from patients with diabetes mellitus and no neurological signs had no effect in our experiments. Despite these objections, a direct role of GAD-Ab is by no means excluded. First, recent work demonstrates that IgG can be internalized by neurons, ${ }^{33}$ making intracellular antigens accessible in experimental settings like ours. Earlier studies already mentioned the ability of IgG to enter neurons. ${ }^{34-35}$ Second, GAD-Ab from patients with or without CNS involvement recognize different epitopes of the GAD protein. ${ }^{3,36}$ Furthermore, GAD-Ab titers are significantly higher in the sera of patients with CNS involvement, some of whom also have evidence of intrathecal synthesis. ${ }^{3}$

Eventually, active immunisation studies with GAD or selective purification of GAD-Ab are required to address the question whether GAD-Ab or other uncharacterized auto-antibodies are responsible for CNS disease. However, production of soluble recombinant GAD is very difficult. It requires large-scale production ${ }^{37}$ and the extraction of $\mathrm{IgG}$ produced by animals immunized with GAD itself remains still a technical challenge. Similarly, further studies will be necessary to understand why some patients develop cerebellar ataxia and others stiff-person syndrome even though they have an apparently identical serological profile. Gathering samples from these rare disorders is essential for these investigations.

\section{Acknowledgments}

We are grateful to John Moore for skillful editing. 
1.Baekkeskov S, Aanstoot HJ, Christgau S et al. Identification of the $64 \mathrm{~K}$ autoantigen in insulindependent diabetes as the GABA-synthesizing enzyme glutamic acid decarboxylase. Nature 1990;347:151-156.

2.Solimena M, Folli F, Denis-Domini S et al. Autoantibodies to glutamic acid decarboxylase in a patient with Stiff-man syndrome, epilepsy, and type 1 diabetes mellitus. New Engl J Med 1988; 318:1012-1020.

3.Honnorat J, Saiz A, Giometto B et al. Cerebellar ataxia with antiglutamic acid decarboxylase antibodies : clinical and immunological study of 14 patients. Arch Neurol 2001; 58: 225-230.

4.Vianello M, Tavolato B, Giometto B. Cerebellar ataxia associated with anti-glutamic acid decarboxylase antibodies. Cerebellum 2003;2:77-79.

5.Dalakas MC, Li M, Fujii M, Jacobowitz DM. Stiff person syndrome: quantification, specificity, and intrathecal synthesis of GAD 65 antibodies. Neurology 2001;57:780-784.

6.Ishida K, Mitoma H, Song S-Y et al. Selective suppression of cerebellar GABAergic transmission by an autoantibody to glutamic acid decarboxylase. Ann Neurol 1999; 46:263-267. 7.Mitoma H, Song S-Y, Ishida K et al. Presynaptic impairment of cerebellar inhibitory synapses by an autoantibody to glutamate decarboxylase. J Neurol Sci 2000; 175:40-44.

8.Oulad Ben Taib N, Manto M, Laute MA, Brotchi J. The cerebellum modulates rodent cortical motor output after repetitive somatosensory stimulation. Neurosurgery 2005; 56:811-820.

9. Oulad Ben Taib N, Manto M, Pandolfo M, Brotchi J. Hemicerebellectomy blocks the enhancement of cortical motor output associated with repetitive somatosensory stimulation. $\mathrm{J}$ Physiol 2005;567:293-300.

10. Oulad Ben Taib N, Laute MA, Pandolfo M, Manto M. Interaction between repetitive stimulation of the sciatic nerve and functional ablation of cerebellar nucleus interpositus in the rat. Cerebellum 2004;3:21-26.

11.Dagonnier M, Laute MA, Pandolfo M, Manto M. Effects of levetiracetam on the production of nitric oxide--an in vivo study. J Neurol 2005;252:727-730.

12.Luft AR, Kaelin-Lang A, Hauser TK et al. Modulation of rodent cortical motor excitability by somatosensory input. Exp Brain Res 2002;142:562-569.

13. Sokolow S, Manto M, Gailly P et al. Impaired neuromuscular transmission and skeletal muscle fiber necrosis in mice lacking Na/Ca exchanger 3. J Clin Invest 2004;113:265-273.

14.Gozariu M, Roth V, Keime F et al. An electrophysiological investigation into the monosynaptic H-reflex in the rat. Brain Res 1998; 782: 343-347.

15.Yamada K, Nabeshima T. Two pathways of nitric oxide production through glutamate receptors in the rat cerebellum in vivo. Neurosci Res 1997;28:93-102.

16.Sommer C, Weishaupt A, Brinkhoff J et al. Paraneoplastic stiff-person syndrome: passive transfer to rats by means of IgG antibodies to amphiphysin. Lancet 2005; 365:1406-1411.

17. Kono S, Miyajima H, Sugimoto M et al.. Stiff-person syndrome associated with cerebellar ataxia and high glutamic acid decarboxylase antibody titer. Intern Med. 2001;40:968-791.

18. Ishida K, Mitoma H, Wada Y et al. Selective loss of Purkinje cells in a patient with antiglutamic acid decarboxylase antibody-associated cerebellar ataxia. J Neurol Neurosurg Psychiatry. Epub 2006 Nov 21.

19. Rakocevic G, Raju R, Semino-Mora C, Dalakas MC. Stiff person syndrome with cerebellar disease and high-titer anti-GAD antibodies. Neurology. 2006;67:1068-1070.

20. Abele M, Weller M, Mescheriakov S et al. Cerebellar ataxia with glutamic acid decarboxylase autoantibodies. Neurology. 1999;52:857-859.

21. Lauria G, Pareyson D, Pitzolu MG, Bazzigaluppi E. Excellent response to steroid treatment in anti-GAD cerebellar ataxia. Lancet Neurol. 2003;2:634-635. 
22.Wall MJ. Endogenous nitric oxide modulates GABAergic transmission to granule cells in adult rat cerebellum. Eur J Neurosci 2003;18:869-878.

23. Contestabile A. Roles of NMDA receptor activity and nitric oxide production in brain development. Brain Res Brain Res Rev 2000;32:476-509.

24.Fedele E, Raiteri M. In vivo studies of the cerebral glutamate receptor/NO/cGMP pathway. Prog Neurobiol 1999;58:89-120.

25.Antzoulatos EG, Byrne JH. Learning insights transmitted by glutamate. Trends Neurosci 2004;27:555-560.

26.Casado M, Isope P, Ascher P. Involvement of presynaptic N-methyl-D-aspartate receptors in cerebellar long-term depression. Neuron 2002;33:123-130.

27.Lev-Ram V, Wong ST, Storm DR, Tsien RY. A new form of cerebellar long-term potentiation is postsynaptic and depends on nitric oxide but not cAMP. Proc Natl Acad Sci USA 2002;99:8389-8393.

28.Jacoby S, Sims RE, Hartell NA. Nitric oxide is required for the induction and heterosynaptic spread of long-term potentiation in rat cerebellar slices. J Physiol 2001;535:825-839.

29.Ciani E, Virgili M, Contestabile A. Akt pathway mediates a cGMP-dependent survival role of nitric oxide in cerebellar granule neurones. J Neurochem $2002 ; 81: 218-228$.

30.Halcak L, Pechanova O, Zigova Z et al. Inhibition of NO synthase activity in nervous tissue leads to decreased motor activity in the rat. Physiol Rev 2000;49:143-149.

31.Sequeira SM, Malva JO, Carvalho AP, Carvahlo CM. Presynaptic NMDA receptor activation inhibits neurotransmitter release through nitric oxide formation in rat hippocampal nerve terminals. Brain Res Mol Brain Res $2001 ; 89: 111-118$.

32. Raju R, Rakocevic G, Chen Z et al. Autoimmunity to GABAA-receptor-associated protein in stiff-person syndrome. Brain. 2006 129:3270-3276.

33.Meneses PI, Zupa-Fernandez A, Maronski M et al. Onconeuronal and control antibodies are internalized by hippocampal neurons resulting in cell death. Neurology 2005, 64 (suppl1) :A52. 34. Fishman PS, Farrand DA, Kristt DA. Internalization of plasma proteins by cerebellar Purkinje cells. J Neurol Sci. 1990;100:43-49.

35. Fishman PS, Farrand DA, Kristt DA. Penetration and internalization of plasma proteins in the human spinal cord. J Neurol Sci. 1991;104:166-175

36.Vianello M, Keir G, Giometto B et al. Antigenic differences between neurological and diabetic patients with anti-glutamic acid decarboxylase antibodies. Eur J Neurol 2005;12:294299.

37. Davis KM, Foos T, Bates CS et al. A novel method for expression and large-scale production of human brain l-glutamate decarboxylase. Biochem Biophys Res Commun. 2000;267:777-82. 
Table 1. Description of the groups of rats investigated.

\begin{tabular}{|c|c|c|}
\hline Experiments & Control Group(s) & Purified IgG fractions / GAD-Ab \\
\hline $\begin{array}{l}\text { Modulation of motor } \\
\text { cortex excitability }^{\mathrm{a}}\end{array}$ & $\begin{array}{l}-\mathrm{n}=6 \text { rats with no } \\
\text { intervention } \\
-\mathrm{n}=6 \text { rats with Ringer } \\
\text { administration }\end{array}$ & $\begin{array}{l}\text {-purified fractions from } 3 \text { patients with } \\
\text { stiff-man syndrome injected in } 4 \text { rats } \\
\text { and purified fractions from } 2 \text { patients } \\
\text { with ataxia injected in } 2 \text { rats (total: } 6 \\
\text { rats) } \\
\text {-GAD-Ab from patients with diabetes } \\
\text { and no neurological complications: } \\
\text { purified fractions from } 3 \text { patients } \\
\text { injected in } 6 \text { rats }\end{array}$ \\
\hline $\mathrm{F} / \mathrm{M}$ ratios $^{\mathrm{b}}$ & $\begin{array}{l}-\mathrm{n}=6 \text { rats with no } \\
\text { administration } \\
-\mathrm{n}=6 \text { rats with } \\
\text { administration of saline }\end{array}$ & $\begin{array}{l}\text {-IgG fractions from } 1 \text { patient with } \\
\text { stiff-man syndrome injected in } 3 \text { rats } \\
\text { and IgG fractions from } 1 \text { patient with } \\
\text { ataxia injected in } 3 \text { rats (total: } 6 \text { rats) } \\
\text {-IgG fractions from } 3 \text { patients with } \\
\text { diabetes and no neurological } \\
\text { complication injected in } 6 \text { rats }\end{array}$ \\
\hline $\begin{array}{l}\text { NMDA-induced } \\
\text { production of nitric oxide } \\
(\mathrm{NO})^{\mathrm{c}}\end{array}$ & $\begin{array}{l}-\mathrm{n}=6 \text { rats with } \\
\text { administration of control } \\
\text { solution }\end{array}$ & $\begin{array}{l}\text {-anti-Yo Ab: purified fractions from } 1 \\
\text { patient injected in } 6 \text { rats; anti-Hu Ab: } \\
\text { purified fractions from } 1 \text { patient } \\
\text { injected in } 6 \text { rats; anti-CV2 Ab: } \\
\text { purified fractions from } 1 \text { patient } \\
\text { injected in } 6 \text { rats } \\
\text {-IgG fractions from } 1 \text { patient with } \\
\text { stiff-man syndrome injected in } 4 \text { rats } \\
\text { and IgG fractions from } 1 \text { patient with } \\
\text { ataxia injected in } 4 \text { rats (total: } 8 \text { rats) }\end{array}$ \\
\hline $\begin{array}{l}\text { Concentrations } \\
\text { glutamate }^{d}\end{array}$ & $\begin{array}{l}-\mathrm{n}=6 \text { rats with } \\
\text { administration of a control } \\
\text { solution }\end{array}$ & $\begin{array}{l}\text {-IgG fractions from } 2 \text { patients with } \\
\text { stiff-man syndrome injected in } 3 \text { rats } \\
\text { and IgG fractions from } 2 \text { patient with } \\
\text { ataxia injected in } 2 \text { rats (total: } 5 \text { rats) }\end{array}$ \\
\hline
\end{tabular}

${ }^{\text {a }}$ Study of the modulation of the excitability of the motor cortex following repetitive stimulation of the contralateral sciatic nerve; ${ }^{\mathrm{b}}$ Study of the ratios $\mathrm{F}$ waves/M response following stimulation of the left tibial nerve and recording in the plantaris muscle; ${ }^{c}$ Measurement of the production of nitric oxide (NO) following administration of NMDA at a concentration of $10 \mathrm{mM}$ via the microdialysis probe; ${ }^{\mathrm{d}}$ Measurements of extra-cellular concentrations of glutamate in microdialysates. See also Methods for the details. 
Control

Baseline

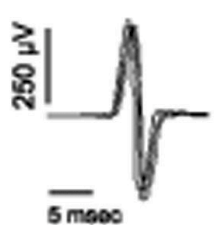

After peripheral stimulation

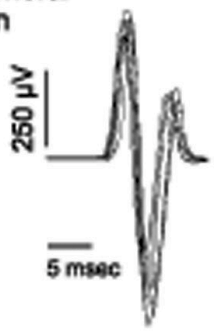

CMAP

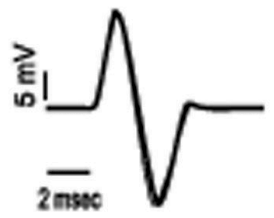

Ringer
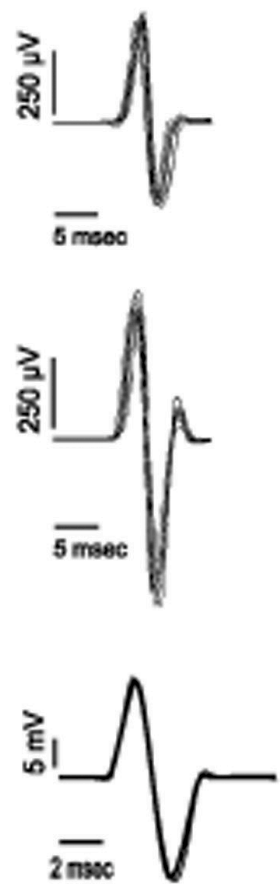

GAD-Ab
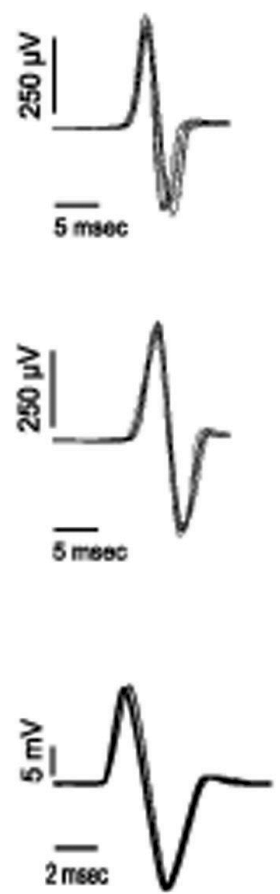

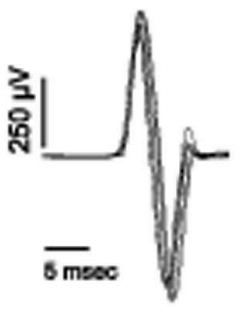

AB-Diabetes
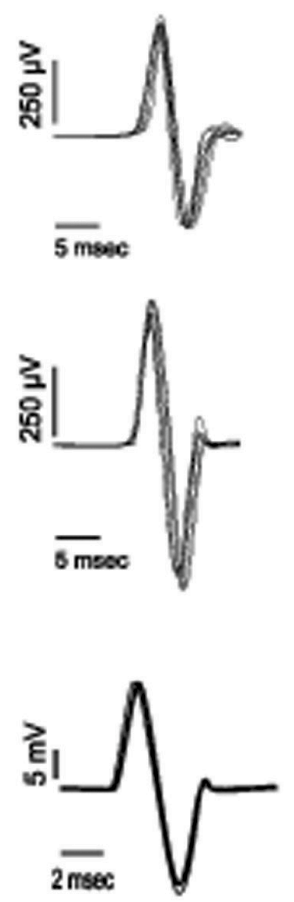

Figure 1A. Modulation of the excitability of the right motor cortex following repetitive somatosensory stimulation of left sciatic nerve. Top traces (superimposition of 8 recordings) correspond to baseline corticomotor potentials. Middle traces correspond to corticomotor responses after repetitive stimulation of the sciatic nerve. Bottom traces correspond to Compound Muscle Action Potentials (CMAPs) before (thin traces) and after (thick traces) repetitive stimulation of the sciatic nerve (duration of stimulation: 1 hour). Left: no cerebellar intervention: the intensity of the corticomotor response is increased following somatosensory stimulation. Middle left: intra-cerebellar administration of Ringer solution: preserved modulation. Middle right: IgG of a patient with stiff-person syndrome and GAD-Ab : micro-injection of IgG blocks the modulation of motor activity. Right. microinjection of IgG from a patient exhibiting diabetes and GAD-Ab without neurological symptom: preservation of the modulation of the excitability of the motor cortex. Recordings in left gastrocnemius muscle, before (baseline) and after intra-cerebellar microinjections ( 5 microL over a period of 5 minutes). 


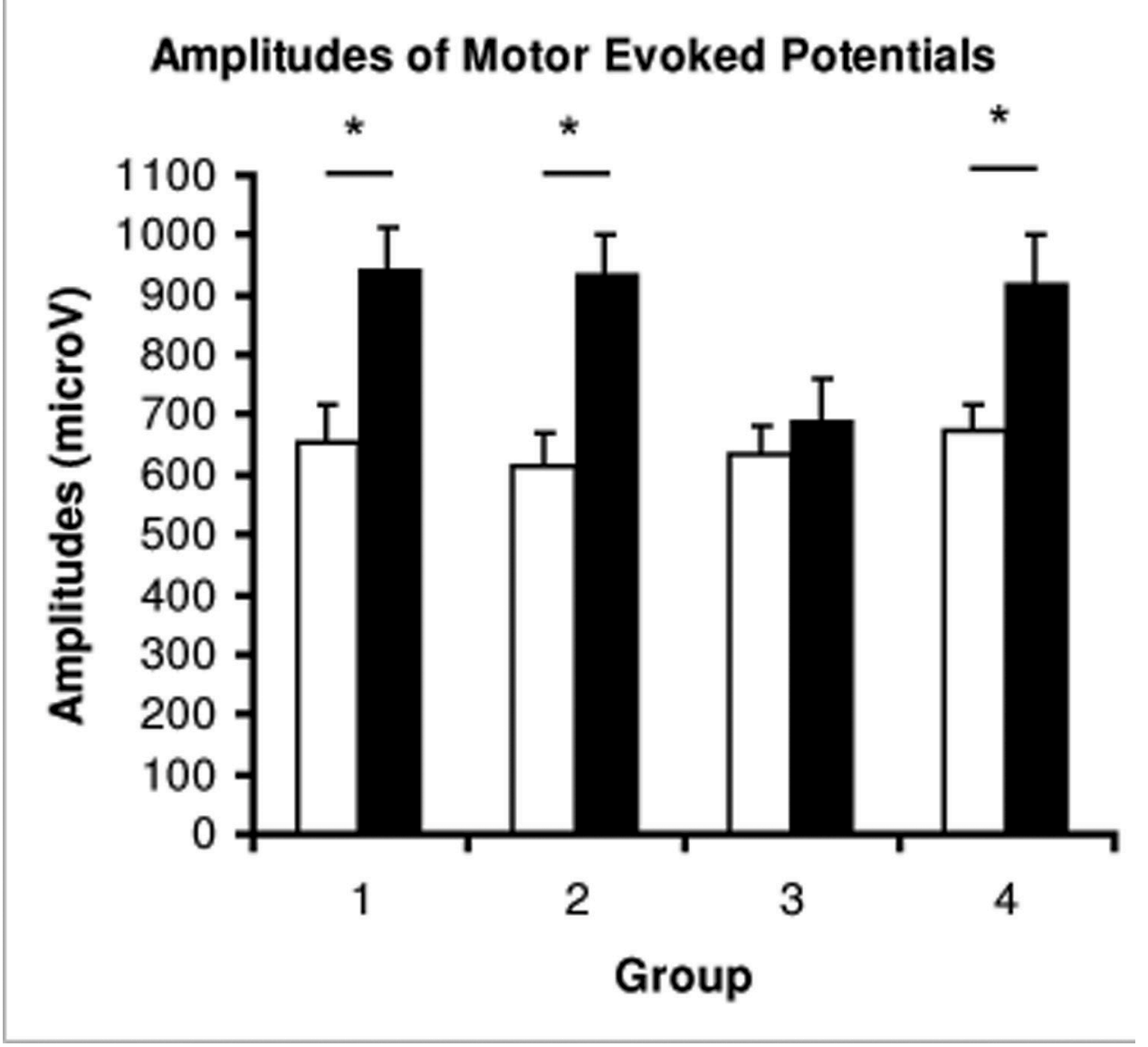

Figure 1B. Means (+/- SD) of amplitudes of motor evoked potentials in the 4 groups of rats. Group 1: no cerebellar intervention ( $\mathrm{n}=6$ rats); Group 2: intra-cerebellar administration of Ringer solution ( $\mathrm{n}=6$ rats); Group 3: intracerebellar administration of GAD-Ab from patients with neurological symptoms $(n=6$ rats: IgG from 3 patients with stiff-person syndrome injected in 4 rats, and $\mathrm{IgG}$ from 2 ataxic patients injected in 2 rats); Group 4: intracerebellar administration of GAD-Ab from patients with diabetes and no neurological symptom (IgG from 3 patients, $\mathrm{n}=6$ rats). Microinjection of a volume of 5 microL of solution administered in groups 2, 3 and $4 . *: p<$ 0.01 . 
A

근

$250 \mathrm{msec}$

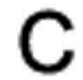

GAD-Ab

극

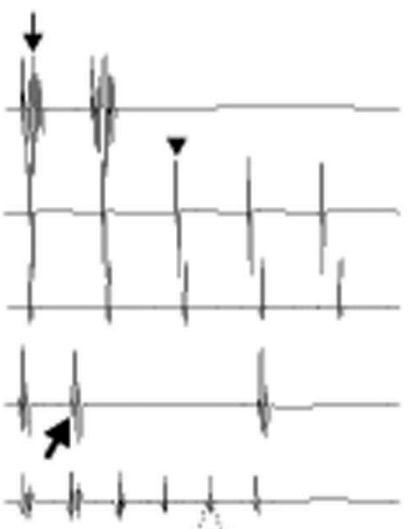

$15 \mathrm{msec}$
B

Ringer

ํㅡㄴ

$250 \mathrm{msec}$

D

Ab-Diabetes

引

$250 \mathrm{msec}$

Figure 2. A. No spontaneous muscle activity detected by needle EMG recordings (left gastrocnemius muscle) in a control rat. B. Para-spinal administration of saline: absence of spontaneous activity (silent trace). C. Administration of IgG from patients with GAD-Ab and stiff-person syndrome (illustrated) or cerebellar ataxia (not shown) induce continuous motor activity (arrow) with repetitive discharges (arrowhead). D. No effect of IgG from patients presenting diabetes without neurological symptoms. Five sites of recordings were explored using a micromanipulator in left gastrocnemius muscle for each rat. 


\section{Mean F/Mean M response}

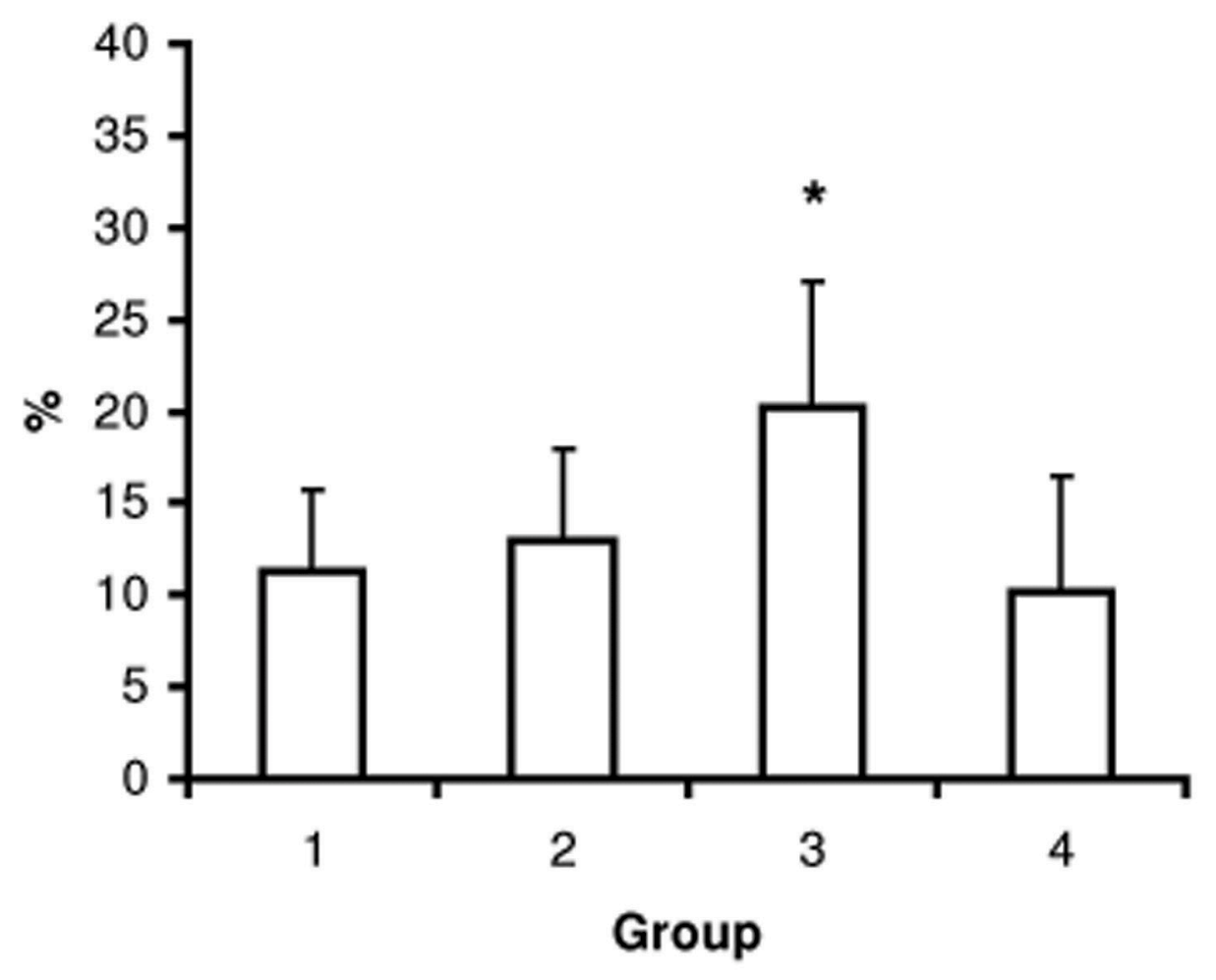

Figure 3. F/M ratios in controls (group 1, 6 rats), in rats receiving saline administration (group 2, 6 rats), in rats receiving IgG from patients with GAD-Ab and stiff-person syndrome or cerebellar ataxia (group 3; IgG from a patient with stiff-person syndrome injected in 3 rats, IgG from a patient with cerebellar ataxia injected in 3 rats), in rats receiving IgG from patients without neurological symptoms (group 4, 3 sera injected in 6 rats). Values obtained in left plantaris muscle 1 hour after lumbar paraspinal administration (10 microL over a period of 10 min). Means (+/- SD) are illustrated. Ratios are expressed in \%. *: $\mathrm{p}<0.05$ 
EFFECTS OF ANTIBODIES ON NMDA-INDUCED PRODUCTION OF NITRIC OXIDE

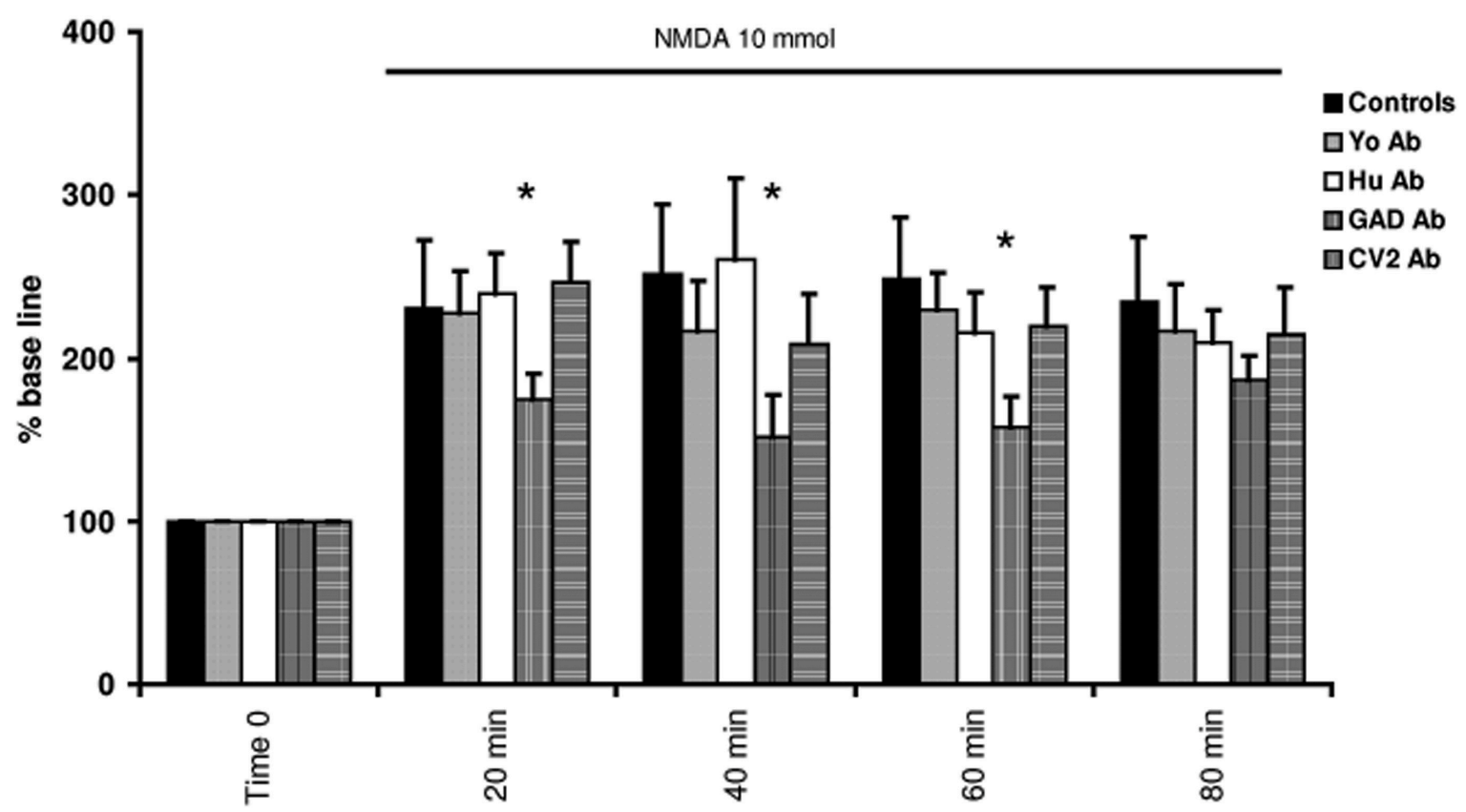

Figure 4. Effects of antibodies on NMDA-induced nitric oxide production in rat cerebellum in vivo. Each bar represents the means (+/-SEM). In addition to a group receiving a control solution, IgG from patients with paraneoplastic cerebellar ataxia (anti-Yo: purified IgG fractions from 1 serum injected in 6 rats, anti-Hu: purified IgG fractions from 1 serum injected in 6 rats, anti-CV2: purified IgG fractions from 1 serum injected in 6 rats) were used as controls. For GAD-Ab, purified IgG fractions from a patient with stiff-person syndrome and from a patient with ataxia were injected in a total of 8 rats (4 rats/each serum). Values are expressed as percentages of basal measurements. $*: \mathrm{p}<0.05$ as compared to the group receiving a control solution or paraneoplastic antibodies. 
EFFECTS OF GAD-Ab ON GLUTAMATE IN CEREBELLAR NUCLEI

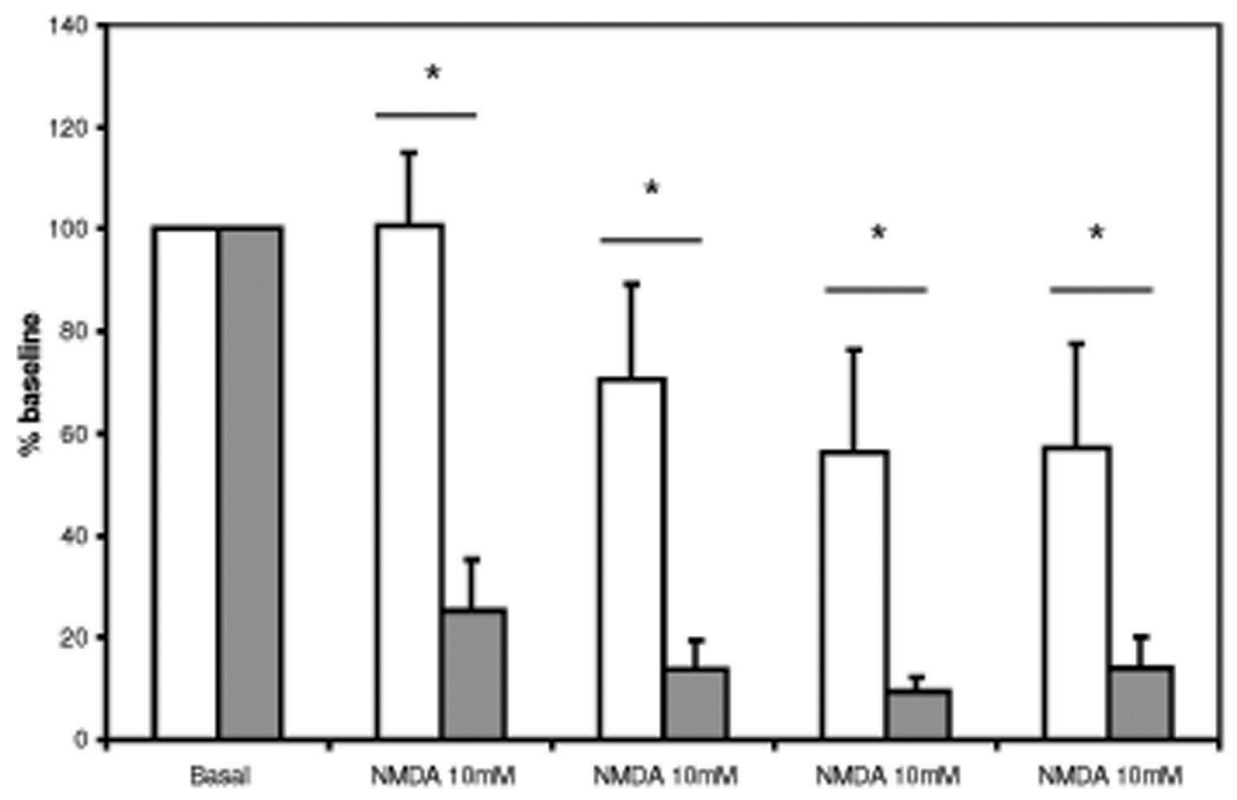

Figure 5. Effects of IgG from patients with GAD-Ab and stiff-person syndrome or cerebellar ataxia on extra-cellular concentrations of glutamate in cerebellar nuclei. Administration of NMDA reduces the contents of glutamate in a control group of 6 rats (grey bars). IgG from patients with GAD-Ab (stiff-person syndrome: 2 sera injected in 3 rats; cerebellar ataxia: 2 sera injected in 2 rats) impair this regulation (white bars; $n=5$ rats). Values are means $+/-\mathrm{SD} *$ : $\mathrm{p}<0.05$. 